\title{
Separation of Isotactic and Syndiotactic Poly(methyl methacrylate) by a Competitive Adsorption Method
}

\author{
Takeaki Miyamoto, Shozo Tomoshige, and Hiroshi InAgaki \\ Institute for Chemical Research, Kyoto University, \\ Uji, Kyoto-fu, 611, Japan.
}

(Received May 15, 1974)

\begin{abstract}
A simple preparative method is presented to separate mixtures of isotactic and syndiotactic poly(methyl methacrylale) (PMMA) into components. The principle of this separation method consists in competitive adsorption of two different tactic polymers from solution onto a solid surface. Separation experiments were carried out at $25^{\circ} \mathrm{C}$ by using nonporous silica gel, Cab-O-Sil, as the adsorbent in chloroform, in which a stereocomplex formation between isotactic and syndiotactic PMMA does not usually occur. Some crystallizable PMMA, designated by Fox, et al., as type-III polymers, were successfully separated into components on a preparative scale by using this method. The result is discussed in terms of the microstructure of these polymer samples.

KEY WORDS Competitive Adsorption / Polymer Separation / Poly(methyl methacrylate) / Stereocomplex / Stereoblock /
\end{abstract}

In a previous paper ${ }^{1}$ we have reported that mixtures of isotactic and syndiotatic poly(methyl methacrylate) (PMMA) can be separated successfully into components with thin layer chromatography (TLC). The TLC technique has been further applied ${ }^{2-4}$ to elucidate the microstructure of crystallizable PMMA, designated by Fox, et al., as type-III polymers, ${ }^{5}$ and it was found that the crystallizable PMMA, which was prepared by using $n$-butylmagnesium chloride as an initiator, was a stereocomplex formed between isotactic and syndiotactic PMMA. ${ }^{2}$ However, further detailed arguments on the condition and mechanism of stereocomplex formation have not yet been made. The main reason was that despite its high utility, TLC was impractical for preparative purposes; it was difficult to obtain sufficient amounts of sample for subsequent characterization experiments.

The present communication describes a new simple method to separate mixtures of isotactic and syndiotactic PMMA into components on a preparative scale. The principle of the separation consists in competitive adsorption of two different tactic polymers from solution onto a solid surface. An attempt is also made to extend the application of this method to separation of the crystallizable type-III PMMA into components.

\section{COMPETITIVE ADSORPTION METHOD}

The idea of this separation method originates from a study reported by $\mathrm{Thies}^{6}$ on adsorption behavior of polystyrene-PMMA mixtures at a solid-liquid interface as well as from two findings of ours. The first is that in the usual TLC in which silica gel is used as adsorbent, chloroform does not allow migration of PMMA, although chloroform is a good solvent for both tactic PMMAs. ${ }^{1}$ This is indicative of the fact that adsorptive interactions between PMMA and silica gel are stronger than those between chloroform and silica. The other is that isotactic PMMA molecules are adsorbed on a silica surface more strongly than those of syndiotactic PMMA. ${ }^{7}$ The above findings allow us to expect that when isotactic PMMA molecules compete with those of syndiotactic PMMA for occupying active sites on silica gel, the former will selectively be adsorbed on silica gel while the latter will be unadsorbed and remain in solution. Thus it may be possible to separate a given mixture of isotactic and syndiotactic PMMA into components. In practice, the separation 
Separation of PMMA by a Competitive Adsorption Method

was carried out by adding silica gel to chloroform solution of isotactic and syndiotactic PMMA mixtures, in which the stereocomplex formation rarely occurs. ${ }^{8}$ A question arising with this experiment is how completely the displacement of syndiotactic PMMA by isotactic PMMA takes place.

\section{EXPERIMENTAL}

\section{Sample Polymers and Characterization}

Fractionated samples of isotactic and syndiotactic-rich PMMA were used in this study. The preparation and the fractionation of these samples have been reported in a previous paper. ${ }^{1}$ The weight-average molecular weight and tacticity of these samples are listed in Table I. To obtain the so-called type-III PMMA, several polymerization runs were made by using $n$ -

Table I. Characterization of sample polymer

\begin{tabular}{lcccc}
\hline \multirow{2}{*}{$\begin{array}{c}\text { Sample } \\
\text { code }\end{array}$} & $M_{w}{ }^{\mathrm{a}}{ }^{\mathrm{a}} \times 10^{-5}$ & \multicolumn{3}{c}{ Triad tacticity } \\
\cline { 3 - 5 } & & $I$ & $H$ & $S$ \\
\hline iMA-3 & 3.95 & 1.0 & - & - \\
iMA-5 & $1.14^{\mathrm{b}}$ & 0.96 & 0.04 & - \\
sMA-2 & 3.40 & 0.04 & 0.16 & 0.80 \\
sMA-4 & 1.53 & - & 0.28 & 0.72 \\
\hline
\end{tabular}

a Weight-average molecular weight determined by equilibrium ultracentrifugation.

b Calculated with viscosity equation $[\eta]=4.8 \times$ $10^{-5} M_{w^{0.8}}$ established for PMMA in chloroform at $25^{\circ} \mathrm{C}$ : J. Bisschoff and V. Desreux, Bull. Soc. Chim. Belg., 61, 10 (1952).
Table II. Preparation conditions of crystallizable type-III polymers

\begin{tabular}{lccrc}
\hline $\begin{array}{l}\text { Polymer } \\
\text { code }\end{array}$ & $\begin{array}{c}\text { Initiator, Monomer, Time, } \\
\text { mol } / l\end{array}$ & mol $/ l$ & min & $\begin{array}{c}\text { Conversion, } \\
\text { wt } \%\end{array}$ \\
\hline cMMA-1 & 0.02 & 0.93 & 20 & 67.0 \\
cMMA-2 $^{\prime}$ & 0.06 & 0.93 & 60 & 31.2 \\
cMMA-3 $^{\text {a }}$ & 0.04 & 1.0 & 240 & 30.0 \\
\hline
\end{tabular}

a This sample was prepared from our previous work, see ref 3 .

butylmagnesium chloride as an initiator in toluene at $-50^{\circ} \mathrm{C}$-as before. ${ }^{2,3}$ Table II summarizes the polymerization data. The products, coded as cMMA-1, cMMA-2, and cMM-3, were subjected to extraction with boiling acetone; ${ }^{9}$ the acetone-insoluble fractions are designated by attaching (i) to the code of each starting product. They were used for subsequent separation experiments.

The NMR spectra were taken with a Hitachi R-20 Spectrometer from 5-\% solution in $\mathrm{CDCL}_{3}$ at room temperature. Triad tacticities were assessed in accordance with the method proposed by Bovey and Tiers. ${ }^{10}$ Table III shows the triad tacticities for three typical products obtained under nearly the same polymerization conditions. The stereoregularity of the products seems to be influenced not only by a slight change in the initiator concentration but also by a delicate difference in the purity of materials used for the polymerization. This problem will be discussed elsewhere.

Intrinsic viscosities $[\eta]$ were determined in chloroform at $25^{\circ} \mathrm{C}$. Results for the characteri-

Table III. Characterization results on acetone-soluble and -insoluble fractions

\begin{tabular}{|c|c|c|c|c|c|}
\hline \multirow{2}{*}{$\begin{array}{l}\text { Polymer } \\
\text { code }\end{array}$} & \multirow{2}{*}{$\begin{array}{l}\text { Acetone } \\
\text { extract, } \%\end{array}$} & \multirow{2}{*}{$\begin{array}{c}{[\eta], \mathrm{d} l / \mathrm{g},} \\
\text { in chloroform, } 25^{\circ} \mathrm{C}\end{array}$} & \multicolumn{3}{|c|}{ Triad tacticity } \\
\hline & & & $I$ & $H$ & $S$ \\
\hline cMMA-1 & & 1.73 & 0.62 & 0.15 & 0.23 \\
\hline Insoluble (cMMA-1i) & 79 & 2.17 & 0.61 & 0.15 & 0.24 \\
\hline Soluble & 21 & 0.28 & 0.64 & 0.21 & 0.15 \\
\hline cMMA-2 & & 0.95 & 0.46 & 0.25 & 0.29 \\
\hline Insoluble (cMMA-2i) & 66 & 1.26 & 0.49 & 0.16 & 0.35 \\
\hline Soluble & 34 & 0.25 & 0.29 & 0.47 & 0.24 \\
\hline cMMA-3 & & & 0.30 & 0.18 & 0.52 \\
\hline Insoluble (cMMA-3i) & 68 & 0.69 & 0.32 & 0.11 & 0.57 \\
\hline Soluble & 32 & 0.44 & 0.26 & 0.34 & 0.40 \\
\hline
\end{tabular}


zation of each fraction are summarized in Table III.

\section{Adsorbent and Solvent}

For adsorption and separation experiments, nonporous silica gel, Cab-O-Sil, M-5 (Cabot Corp., Boston, Massachusetts) was used, which has a surface area of $175-220 \mathrm{~m}^{2} / \mathrm{g}$ determined by adsorption of nitrogen. Just before use, the adsorbent was activated at $110^{\circ} \mathrm{C}$ for $24 \mathrm{hr}$. The chloroform used as solvent was carefully purified according to the standard procedure.

\section{Adsorption Isotherms}

Adsorption experiments were carried out in sealed glass ampoules containing a known weight of adsorbent and $5 \mathrm{ml}$ of each polymer solution with different initial concentrations. The sealed glass ampoule was then placed in a constant temperature bath at $25^{\circ} \mathrm{C}$ and agitated. The supernatant was subsequently separated from the adsorbent by a preparative centrifuge. The amount of adsorbed polymer was determined with a Perkin-Elmer Model 521 IR Spectrometer, by measuring the change in the adsorption of ester carbonyls $\left(1723 \mathrm{~cm}^{-1}\right)$ contained in the supernatant. This adsorption is independent of the tacticity, and the peak height is related to polymer concentration by calibrating with solutions of known concentrations.

The time required to attain the adsorption equilibrium was determined according to our preliminary result obtained for stactic PMMA. ${ }^{11}$ The rate of adsorption from its chloroform solution onto silica gel was very fast, and an adsorption equilibrium appeared to be attained after a time ranging from 0.5 to $2 \mathrm{hr}$. However, prolonging the time of observation, we found that this equilibrium was merely apparent and the polymer amount adsorbed at this stage began to decrease very slowly; the true equibrium was finally attained after about $20 \mathrm{hr}$. The reason is still open for discussion. However, the difference between the polymer amounts adsorbed at the apparent and true equilibrium was as small as $5 \%$ so that the polymer amount adsorbed after $2 \mathrm{hr}$ could be regarded as that at the true equilibrium.

Figure 1 shows equilibrium adsorption isotherms for isotactic(iMA-3) and syndiotacticrich PMMA(sMA-2) in chloroform solution at $25^{\circ} \mathrm{C}$. The amounts of polymer adsorbed per gram of Cab-O-Sil $(\mathrm{mg} / \mathrm{g})$ are plotted against the equilibrium concentration of polymer remaining in solution $(\mathrm{g} / \mathrm{d} l)$. Both equilibrium adsorption isotherms are characterized by a steep initial slope and plateau. The levels of adsorption at each plateau, namely, the maximum amounts of the adsorbed isotactic and syndiotactic-rich PMMA were approximately 120 and $105 \mathrm{mg}$ per unit gram of Cab-O-Sil, respectively. It is obvious that the difference between these amounts is due not to the difference in

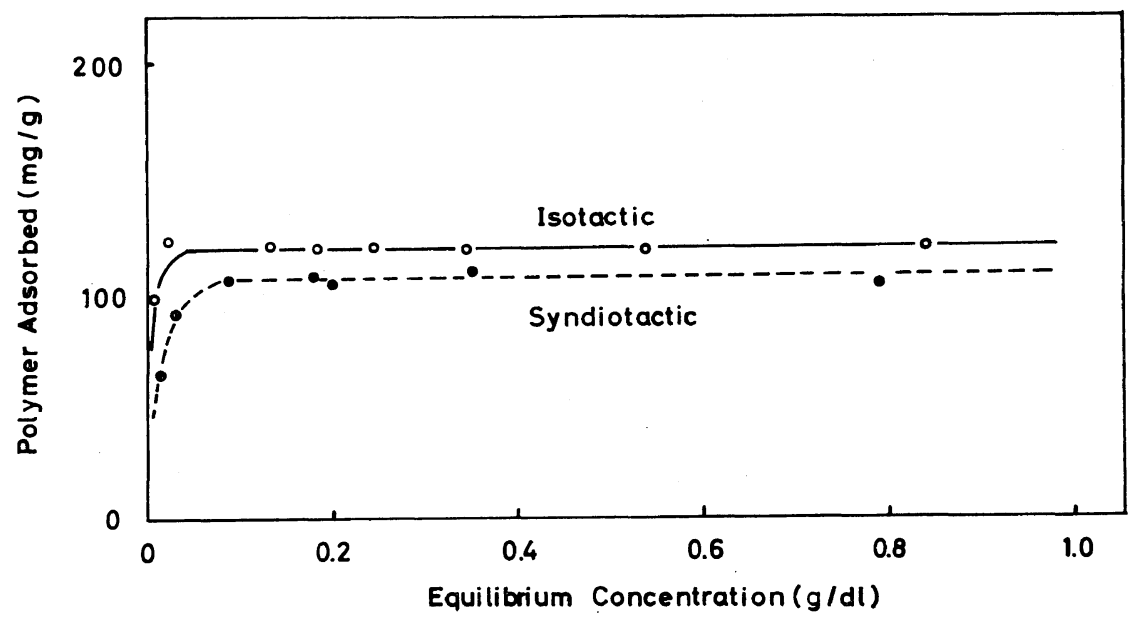

Figure 1. Equilibrium adsorption isotherms of isotactic and syndiotactic PMMA on silica gel from chloroform solution at $25^{\circ} \mathrm{C}$ : $(\bigcirc)$, isotactic; (O), syndiotactic PMMA. 
molecular weight but to the difference in the type of stereoregularity, since each sample polymer could be regarded from previous TLC experiment $^{12}$ as a fraction with a fairly low heterogeneity in moleculuar weight and the present experiment was conducted at nearly the same molecular weight level.

\section{Separation Technique}

Separation experiments were carried out in sealed glass ampoules. A mixture of iMA-5 and sMA-4 (1:1 by weight) was dissolved in chloroform at a concentration of $0.5 \mathrm{~g} / \mathrm{d} l . \quad 100$ $\mathrm{m} l$ of this polymer solution was shaken for $2 \mathrm{hr}$ at $25^{\circ} \mathrm{C}$ with Cab-O-Sil of a fixed weight, which was calculated on the basis of the equilibrium amount of adsorption found for isotactic PMMA, i.e., $120(\mathrm{mg} / \mathrm{g})$. The supernatant was then separated from the adsorbent by centrifugation. The unadsorbed polymer remaining in solution was obtained from the supernatant by evaporation. The polymer adsorbed on the silica gel was recovered by extraction with an acetone-chloroform mixture ( $1: 1$ by volume) at the boiling point, followed by evaporation of the solvent. Both sample polymers were purified by precipitating them from benzene solution into methanol, after the benzene solutions had been filtrated with a glass filter. Weight recovery of samples by this procedure was approximately $95 \%$.

\section{RESULTS AND DISCUSSION}

\section{Separation of a Mixture of Isotactic and Syndiotactic PMMA}

In the present work, no equilibrium adsorption isotherm for a mixture of isotactic and syndiotactic PMMA was studied. However, we have investigated to what extent the displacement of syndiotactic PMMA by isotactic PMMA on a silica gel surface takes place. For this purpose, a series of preliminary experiments on a 1:1 mixture of isotactic and syndiotactic-rich PMMA have been carried out to see whether or not one still finds the syndiotactic molecules being adsorbed even after the adsorption has been equilibrated between the isotactic molecules and the silica gel. In practice, adsorption experiments on the polymer mixture were made in the presence of Cab-O-Sil of various amount relative to that of the isotactic polymer present. As described previously, the amount of equilibrium adsorption found for the isotactic polymer was $120(\mathrm{mg} / \mathrm{g})$. Taking this result into consideration, we have added Cab-O-Sil in the amount of $90 \%$ of the equilibrium value for the first run of the experiments, $110 \%$ for the secoud run, and $150 \%$ for the last run.

Figure $2 \mathrm{a}$ shows the $90-\mathrm{MHz}$ NMR spectrum for the fraction recovered from the supernatant obtained by the first run; Figures $2 b$ and $2 c$ show those for the fractions resulting from the second and third run, respectively. From this result it is seen that the isotactic polymer no

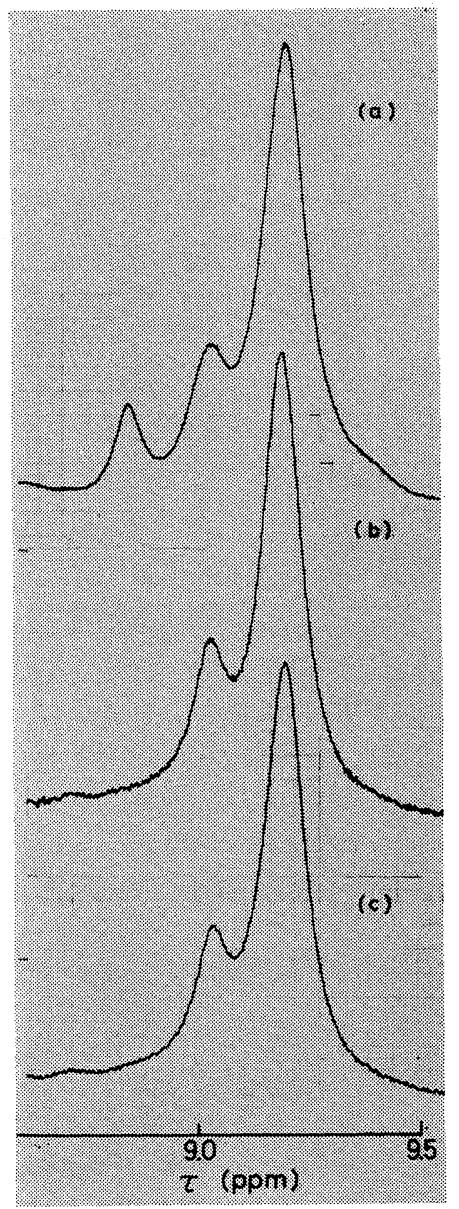

Figure 2. $90-\mathrm{MHz}$ NMR spectra of the unadsorbed fractions obtained by addition of Cab-O-Sil in the amount of $90 \%$ of the equilibrium value (2a), $110 \%(2 \mathrm{~b})$, and $150 \%(2 \mathrm{c})$. 
longer exists in the supernatant when the amount of Cab-O-Sil added exceeds that for the equilibrium by a little bit. The syndiotacticities assessed from the spectra given by Figures $2 b$ and $2 \mathrm{c}$ are in good coincidence with that for the component syndiotactic-rich sample. However, the isotactic polymer recovered from the adsorbed state, which corresponds to the result shown by Figure 2a, indicated the presence of a very small amount of the syndiotactic-rich component. This result may be attributed to the experimental difficulty that is encountered in removing the supernatant completely from the adsorbent. The separation mechanism can be

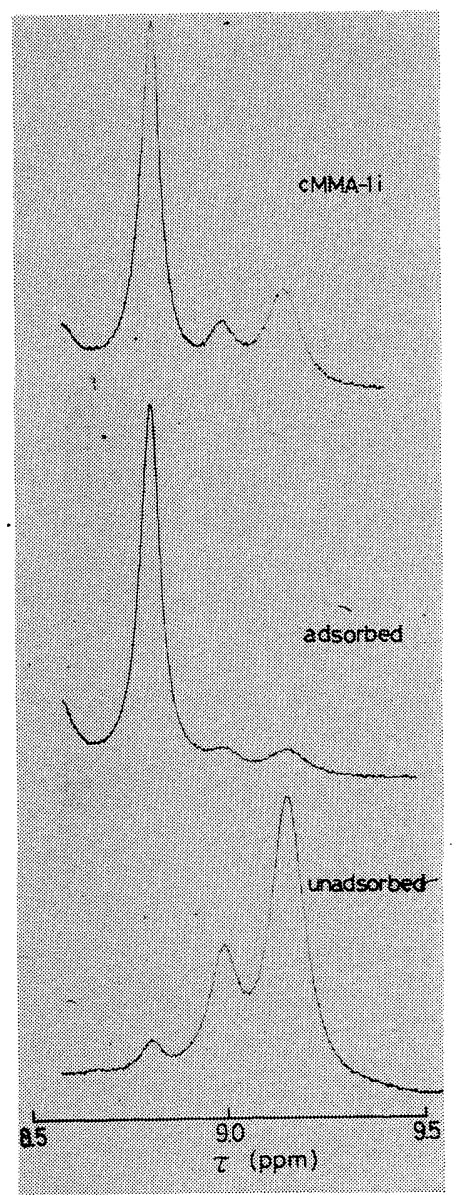

Figure 3. $90-\mathrm{MHz}$ NMR spectra of the acetoneinsoluble fraction, cMMA-1i, and the species involved in the adsorbed and the unadsorbed portion separated from this fraction after competitive adsorption. understood well in terms of our theory. ${ }^{1,7}$ Separation of Crystallizable Type-III PMMA

Next, we have applied this separation method to crystallizable type-III polymers to investigate the microstructure of these polymers. According to the same procedure described above, three typical acetone-insoluble fractions were separated into adsorbed and unadsorbed portions, and subsequently these fractions were recovered. In calculating the amount of Cab-O-Sil to be added it is necessary to estimate the content of the isotactic component in the sample. Such an estimate is very important to obtain a sharp fraction of either tactic component, as anticipated

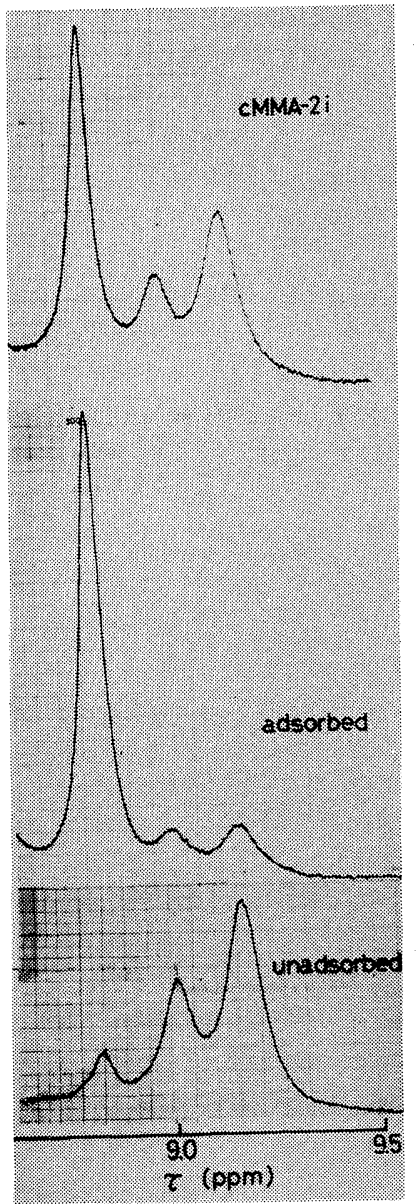

Figure 4. $90-\mathrm{MHz}$ NMR spectra of the acetoneinsoluble fraction, cMMA-2 $i$, and the species involved in the adsorbed and the unadsorbed portion separated from this fraction after competitive adsorption. 
from the result given in Figure 2a. For the purpose, we have assumed that the sample is a mixture of isotactic and syndiotactic-rich PMMA and that the triad isotacticity found for the sample results only from the isotactic component. In other words, the isotactic triad due to the syndiotactic-rich component was assumed to be negligible.

Figure 3 shows the NMR spectra for sample cMMA-1i and its component species obtained from the adsorbed and unadsorbed states. Figures 4 and 5 indicate the same information for the other samples, cMMA-2i and cMMA-3i. The triad tacticities assessed from the NMR

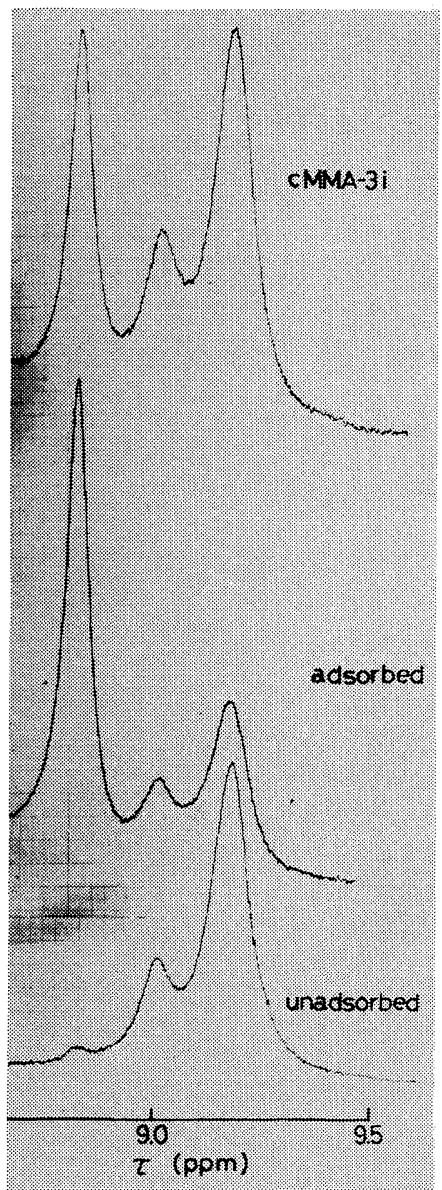

Figure 5. $90-\mathrm{MHz}$ NMR spectra of the acetoneinsoluble fraction, cMMA-3i, and the species involved in the adsorbed and the unadsorbed portion separated from this fraction after competitive adsorption.
Table IV. Triad tacticities of acetone-insoluble fractions of starting PMMA and two portions obtained after competitive adsorption

\begin{tabular}{lccc}
\hline \multirow{2}{*}{$\begin{array}{l}\text { Polymer } \\
\text { code }\end{array}$} & \multicolumn{3}{c}{ Triad tacticity } \\
\cline { 2 - 4 } & $I$ & $H$ & $S$ \\
\hline cMMA-1i & 0.61 & 0.15 & 0.24 \\
Adsorbed & 0.84 & 0.08 & 0.08 \\
Unadsorbed & 0.09 & 0.27 & 0.64 \\
\hline cMMA-2i & 0.49 & 0.16 & 0.35 \\
Adsorbed & 0.77 & 0.12 & 0.11 \\
Unadsorbed & 0.13 & 0.31 & 0.56 \\
\hline cMMA-3i & 0.32 & 0.11 & 0.57 \\
Adsorbed & 0.58 & 0.14 & 0.28 \\
Unadsorbed & 0.05 & 0.24 & 0.71 \\
\hline
\end{tabular}

spectra are summarized in Table IV.

The spectra shown in Figures 3 and 4 imply that the separations of cMMA-1i and cMMA-2 $i$ into an isotactic and a syndiotactic-rich component are almost perfect, but every spectrum obtained for each isotactic component is indicative of a low content of syndiotactic triads. Such an appearance of syndiotactic triads should be studied from different aspects. The first aspect may concern some steric irregularity which the isotactic component possesses in situ. However, we have no means to investigate this aspect. The other aspect may be related to a question whether the separated isotactic component is adulterated with the syndiotactic-rich component.

The same TLC technique employed previous$1 y^{1-3}$ can be applied to the above problem. We used ethyl acetate as the developing agent, which can develop only the syndiotactic (or stactic) PMMA and allows the isotactic PMMA to remain immobile on the starting line. The TLC chromatogram indicated that the isotactic component of sample cMMA-3i was separated into an immobile component and another component which reached the proximity of the solvent front, the latter was assigned to the syndiotactic-rich component. Such adulteration may originate from various sources, such as the adsorptive equilibrium between the isotactic and syndiotactic components, the addition of an inappropriate amount of adsorbent, the difficulty in removal of supernatant from adsorbent, and 
so forth. Whatever is most dominant among these factors, the essential thing is to liberate perfectly the syndiotactic-rich component present as the adulterant from the isotactic component in order to determine its microstructure. Such a separation experiment with TLC is in progress in our laboratory.

Despite the presence of unexplainable factors mentioned above, our result yields evidence to show that these crystallizable type-III PMMA are merely mixtures of isotactic and syndiotacticrich polymers. This supports our previous conclusion $^{3}$ that the essential step in polymerization of this type may be conditioned by the stereocomplex formation.

\section{REFERENCES}

1. H. Inagaki, T. Miyamoto, and F. Kamiyama, J. Polym. Sci., Part B-7, 329 (1969).

2. T. Miyamoto and H. Inagaki, Macromolecules,
2, 554 (1969).

3. T. Miyamoto and H. Inagaki, Polymer J., 1, 46, (1970).

4. R. Butter, Y. Y. Tan, and G. Challa, Polymer, 14, 171 (1973).

5. T. G. Fox, B. S. Garrett, W. E. Goode, S. Gratch, J. F. Kincaid, A. Spell, and J.D. Stroupe, J. Amer. Chem. Soc., 80, 2341 (1958).

6. C. Thies, J. Phys. Chem., 70, 3783 (1966).

7. T. Miyamoto and H.-J. Cantow, Makromol. Chem., 162, 43 (1972).

8. W. B. Van Den Berg, B. Hijmans, P. Piet, and D. Heikens, Nature, 217, 949 (1968).

9. A. M. Liquori, G. Anzuino, M. D'Alagni, P. DeSantis, and M. Savino, Nature, 206, 358 (1965).

10. F. A. Bovey and G. V. D. Tiers, J. Polym. Sci., 44, 173 (1960).

11. T. Miyamoto, S. Tomoshige, and H. Inagaki, Makromol. Chem., in press.

12. H. Inagaki, F. Kamiyama, and T. Yagi, Macromolecules, 4, 133 (1971). 